\title{
Non-Euclidean phasor analysis for quantification of oxidative stress in ex vivo human skin exposed to sun filters using fluorescence lifetime imaging microscopy
}

Sam Osseiran

Elisabeth M. Roider

Hequn Wang

Yusuke Suita

Michael Murphy

David E. Fisher

Conor L. Evans 


\title{
Non-Euclidean phasor analysis for quantification of oxidative stress in ex vivo human skin exposed to sun filters using fluorescence lifetime imaging microscopy
}

\author{
Sam Osseiran, ${ }^{\mathrm{a}, \mathrm{b}}$ Elisabeth M. Roider, ${ }^{\mathrm{c}}$ Hequn Wang, ${ }^{\mathrm{a}}$ Yusuke Suita,,${ }^{\mathrm{c}}$ Michael Murphy, ${ }^{\mathrm{a}}$ David E. Fisher, ${ }^{\mathrm{c}}$ and \\ Conor L. Evans ${ }^{a, *}$ \\ aHarvard Medical School, Massachusetts General Hospital, Wellman Center for Photomedicine, Charlestown, Massachusetts, United States \\ bMassachusetts Institute of Technology, Harvard-MIT Division of Health Sciences and Technology, Cambridge, Massachusetts, United States \\ 'Harvard Medical School, Massachusetts General Hospital, Cutaneous Biology Research Center, Charlestown, Massachusetts, United States
}

\begin{abstract}
Chemical sun filters are commonly used as active ingredients in sunscreens due to their efficient absorption of ultraviolet (UV) radiation. Yet, it is known that these compounds can photochemically react with UV light and generate reactive oxygen species and oxidative stress in vitro, though this has yet to be validated in vivo. One label-free approach to probe oxidative stress is to measure and compare the relative endogenous fluorescence generated by cellular coenzymes nicotinamide adenine dinucleotides and flavin adenine dinucleotides. However, chemical sun filters are fluorescent, with emissive properties that contaminate endogenous fluorescent signals. To accurately distinguish the source of fluorescence in ex vivo skin samples treated with chemical sun filters, fluorescence lifetime imaging microscopy data were processed on a pixel-by-pixel basis using a non-Euclidean separation algorithm based on Mahalanobis distance and validated on simulated data. Applying this method, ex vivo samples exhibited a small oxidative shift when exposed to sun filters alone, though this shift was much smaller than that imparted by UV irradiation. Given the need for investigative tools to further study the clinical impact of chemical sun filters in patients, the reported methodology may be applied to visualize chemical sun filters and measure oxidative stress in patients' skin. ๑ 2017 Society of Photo-Optical Instrumentation Engineers (SPIE) [DOI: 10.1117/1.JBO.22.12.125004]
\end{abstract}

Keywords: fluorescence lifetime imaging microscopy; two-photon excitation fluorescence; nicotinamide adenine dinucleotides; flavin adenine dinucleotides; sun filters; oxidative stress.

Paper 170555R received Aug. 23, 2017; accepted for publication Nov. 20, 2017; published online Dec. 9, 2017.

\section{Introduction}

Despite the growing use of sunscreen products, skin cancer incidence steadily remains on the rise, with one in five Americans expected to develop some form of skin cancer throughout their life. ${ }^{1,2}$ Furthermore, while some evidence of sunscreen protection against melanoma has been obtained, ${ }^{3}$ this was rather modest and challenging to demonstrate, in comparison to other cutaneous tumors. ${ }^{4}$ One potential explanation for this discrepancy involves the chemical sun filters in sunscreens, which are responsible for absorbing ultraviolet (UV) radiation to minimize DNA damage to underlying skin cells. Prior studies have shown that some commercial chemical sun filters are capable of generating reactive oxygen species (ROS) when exposed to UV radiation under in vitro experimental conditions. ${ }^{5}$ Consequently, it has been proposed that these compounds may generate ROS in vivo at the site of application, thereby inducing oxidative stress in skin. ${ }^{5,6}$ Over a prolonged period of time, chronic oxidative stress may in turn contribute to skin aging, actinic keratosis, DNA damage, as well as the onset and pathogenesis of squamous and basal cell carcinomas. ${ }^{7-10}$

The study of oxidative stress in biological samples can be carried out using a wide variety of techniques. Traditional tissue

*Address all correspondence to: Conor L. Evans, E-mail: evans.conor@mgh .harvard.edu processing methods can be used to extract, isolate, and quantify the amount of flavin and nicotinamide adenine dinucleotides (FAD and NADH, respectively), two key coenzymes in redox metabolism, using western blotting, quantitative polymerase chain reaction, immunohistochemistry, or liquid chromatography/tandem mass spectrometry. ${ }^{11,12}$ However, the processing necessary for these techniques is either inherently destructive or does not provide spatial information, and as such, bars the ability to assess heterogeneous cellular-level responses within the tissue. On the other hand, exogenous labels, such as fluorogenic probes, can be used to preserve spatial integrity and have had great success mapping spatial patterns of ROS generation in vitro..$^{5}$ These dyes, however, can be challenging when used in skin, whether ex vivo or in vivo, as many probes experience uneven uptake within skin. ${ }^{13}$ This irregular diffusion leads to regional differences in probe signal intensity that may arise primarily due to uneven distribution rather than true contrast in oxidative stress.

Given these challenges, label-free optical imaging techniques have been found to be well-suited for probing oxidative stress within tissues. ${ }^{14}$ These tools capture fluorescence signals that arise from metabolic coenzymes of interest, enabling the mapping of oxidative stress on the subcellular level with submicron

1083-3668/2017/\$25.00 @ 2017 SPIE 
spatial resolution throughout intact tissue. Label-free imaging also removes any artifacts that can result from processing and permeabilizing tissue, as the imaging contrast arises from endogenous fluorescence rather than exogenous reporters of ROS.

To study oxidative stress within skin without such exogenous labels, one advantageous approach is two-photon excitation fluorescence (TPEF) microscopy that directly assesses the fluorescence intensities of FAD and NADH. ${ }^{12,15-17}$ The intrinsic fluorescence of these metabolic coenzymes is advantageous for studying cellular metabolic perturbations, as FAD only fluoresces in its oxidized form, while NADH is strictly fluorescent in its reduced form; their counterparts, $\mathrm{FADH}_{2}$ and $\mathrm{NAD}^{+}$, are not fluorescent. Together, these coenzymes act as electron carriers during the catabolic processes that result in ATP production, and the relative oxidized-to-reduced coenzyme ratio, commonly referred to as the redox ratio, can be used to make inferences about a cell's metabolic state. By imaging these intrinsic fluorophores within a given biological sample, one can then compare the signals obtained from both molecules to quantitatively assess oxidative stress using the ratio of FAD fluorescence intensity to that of NADH, i.e., FAD/NADH. ${ }^{14}$ The normalized optical redox ratio (NORR), defined as FAD/ $(\mathrm{NADH}+\mathrm{FAD})$, is also a commonly used imaging metric of oxidative stress, as it is strictly bound between 0 and 1 and more closely follows a normal distribution. ${ }^{12,18}$

This study sought to devise a method to noninvasively and nondestructively examine the impact of chemical sun filter exposure on the redox metabolism of epidermal skin cells and determine whether these compounds can cause an oxidative shift in skin tissue. Viable, cultured ex vivo models of human skin were imaged using TPEF microscopy to quantify the change in optical redox ratio on a cell-to-cell level throughout the epidermis of skin based on the endogenous fluorescence of FAD and NADH. ${ }^{14} \mathrm{~A}$ minimalist model formulation of sunscreen was designed for the present study containing five commonly used chemical sun filters in proportions that emulate a sun protection factor (SPF) of roughly 45 . This minimalist approach was preferred to avoid any potential interference from inactive sunscreen ingredients while adhering to FDA guidelines on maximum concentrations for each individual sun filter. ${ }^{19}$

Due to the competing fluorescence contribution of sun filter compounds, however, an analysis method based on fluorescence lifetime imaging microscopy (FLIM) and phasor analysis had to be developed to extract the optical redox ratio and map oxidative stress changes within skin. Indeed, the fluorescence from sunscreen formulations is not only fairly weak but also varies depending on the combination and relative concentrations of chemical sun filters therein. ${ }^{20}$ In turn, this variability limits the use of hyperspectral fluorescence imaging to provide accurate and reliable contrast between endogenous and exogenous sources of fluorescence. In contrast, the photophysical dynamics of these sun filters is consistently short-lived, ${ }^{10,21}$ warranting a highly sensitive time-correlated approach, such as FLIM, to accurately decouple the endogenous tissue fluorescence from that of the exogenous compounds based on differences in fluorescence lifetime. This method successfully enabled the quantification of oxidative stress caused by both UV light exposure and sun filters and additionally allowed for the direct imaging of sun filters as they diffused through skin tissue.

It is worth noting that TPEF imaging is not exclusive to $\mathrm{NADH}$ and FAD, as some other endogenous compounds in skin exhibit fluorescent properties as well, including melanin, elastin, collagen, and keratin. Conveniently, the optimal excitation and emission parameters for probing melanin autofluorescence are distinct from those of NADH and FAD; moreover, the signal from these coenzymes dominates the autofluorescence from the stratum spinosum layer of the epidermis. ${ }^{22}$ As for fluorescent extracellular proteins, elastin and collagen are primarily found within the dermis while keratin forms within the more superficial layers of the epidermis. As such, the imaging parameters described in the present study are based on commonly used methods to maximize signals from NADH and FAD within the stratum spinosum roughly $50 \mu \mathrm{m}$ below the skin surface. ${ }^{11,12,18,23}$

\section{Materials and Methods}

\subsection{Sun Filter Formulation}

The minimalist model formulation of sunscreen was composed of five commonly used chemical sun filters (avobenzone, octocrylene, homosalate, octisalate, and oxybenzone) dissolved in a lipophilic solvent typically included in sunscreen formulations (C12-15 alkyl benzoate, also known as Finsolv TN) in proportions that emulate an SPF of roughly 45 (see Table 1). The fluorescence of the formulation is primarily attributed to the presence of avobenzone, one of the most commonly used chemical sun filters in sunscreens. ${ }^{10,24}$

\subsection{Tissue Culture and Processing}

To study oxidative stress in human skin in response to chemical sun filters, discarded skin tissues were obtained from elective patient surgeries in compliance with the institutional review board of Massachusetts General Hospital (MGH IRB protocol \#2014P00135). The skin was then processed on arrival, where it was cut into square pieces roughly 1 to $2 \mathrm{~cm}^{2}$ in size, placed on a nutritive gel bed made of complete Roswell Park Memorial Institute (RPMI) medium with 2\% agarose, and maintained in an incubator at $37^{\circ} \mathrm{C}$ with $5 \%$ atmospheric $\mathrm{CO}_{2}$. Ex vivo human skin explants were imaged using FLIM under four different experimental conditions: treatment with a $30-\mu \mathrm{L}$ topical dose of either the sun filter formulation described in Table 1 or Finsolv TN alone as a vehicle-only control and subsequently, immediately exposed to either 0 or $20 \mathrm{~J} / \mathrm{cm}^{2}$ of UVA irradiation. This corresponds to 1 minimal erythemal dose (MED) for a fair-skinned individual of Fitzpatrick skin type I, i.e.,

Table 1 Composition of sun filter formulation.

\begin{tabular}{lc} 
Compound & Concentration $(\% \mathrm{~m} / \mathrm{m})$ \\
\hline Avobenzone & 3 \\
Octocrylene & 4 \\
Homosalate & 8 \\
Octisalate & 5 \\
Oxybenzone & 4 \\
Finsolv TN & 76 \\
\hline
\end{tabular}


the minimum amount of UVA irradiation necessary to elicit a reddening response in skin. ${ }^{25,26}$ Three distinct areas were then imaged for each skin sample, where each field of view consisted of a representative slice of the stratum spinosum within the viable epidermis, roughly $50 \mu \mathrm{m}$ below the skin surface.

\subsection{TPEF and FLIM Imaging}

In using a fluorescence-based approach to study the effects of exogenous compounds, such as chemical sun filters, a common challenge often arises: many of these compounds are natively fluorescent ${ }^{20}$ and may, therefore, interfere with the fluorescent signals from NADH and/or FAD. In the case of this study, it was found that the mixture of chemical sun filters was itself fluorescent, with excitation and emission properties similar to those of NADH. The formulation was not found to be emissive in the FAD spectral range. Because of this fluorescence, optical redox ratio measurements cannot be obtained on tissue exposed to the formulation, since there is no way to distinguish the fluorescence signal of the sun filters from that of NADH using TPEF microscopy alone. To circumvent this obstacle, the sun filter formulation was imaged using two-photon FLIM to determine if its fluorescence can be distinguished from that of NADH based on lifetime.

The laser source used to perform TPEF imaging is a dual-output femtosecond pulsed laser system (Spectra-Physics Insight DeepSee), where one output can be tuned to any integer wavelength ranging from 680 to $1300 \mathrm{~nm}$. The beam was directed into the input port of a commercial inverted microscope (Olympus FV1000) for imaging with a $60 \times$ water immersion objective (Olympus UPLSAPO 60XW, 1.20 NA), and the total laser power at the output of the objective was below $35 \mathrm{~mW}$ for all imaging experiments. The fluorescence signals were detected using two distinct photomultiplier tubes (Hamamatsu H7422P-40 for NADH and H7422P-50 for FAD). The FLIM data were collected using time-correlated single-photon counting hardware (Becker \& Hickl SPC-150), with each image containing $256 \times 256$ pixels in area and 256 time bins for each pixel's associated fluorescence decay trace. The imaging acquisition parameters resulted in photon count rates ranging from $2 \times 10^{4}$ to $2 \times 10^{5}$ photons per second, with a total acquisition period of $90 \mathrm{~s}$. The calibration of the FLIM system was ensured using a solution of fluorescein ( $\mathrm{pH}$ 9.0) with a known lifetime of 4.05 ns. Each area was first imaged with 755-nm laser light for multiphoton excitation of NADH with detection of fluorescence from 445 to $480 \mathrm{~nm}$, followed by 860 -nm excitation for FAD with emission detection from 500 to $550 \mathrm{~nm}$.

\subsection{CARS Imaging}

The Finsolv TN molecule can be readily visualized using coherent anti-Stokes Raman scattering (CARS) microscopy by probing the symmetric $\mathrm{CH}_{2}$ stretching vibration at $2845 \mathrm{~cm}^{-1}$ of its long C12-15 hydrocarbon tail. As such, the tunable output of the laser source was set to $803 \mathrm{~nm}$ as the pump beam and the fixed 1040-nm output was used as the Stokes beam to generate a strong anti-Stokes signal at $654 \mathrm{~nm}$, allowing for the monitoring of solvent penetration dynamics in skin. To this aim, an ex vivo skin sample was given a single topical treatment of Finsolv TN and, subsequently, monitored at 15-min intervals over the course of $2 \mathrm{~h}$, examining depths 0,40 , and $80 \mu \mathrm{m}$ below the skin surface.

\subsection{Non-Euclidean Phasor Analysis}

The separation of fluorescent species based on their lifetime is best achieved using the phasor approach. ${ }^{18,27-29}$ This method is derived from Fourier analysis, where rather than fitting a linear combination of decaying exponentials onto a decay trace $I_{i j}(t)$ in the temporal domain, the data are transformed into a phasor with coordinates $\left(G_{i j}, S_{i j}\right)$ defined as

$$
\begin{gathered}
G_{i j}=\frac{\int_{0}^{\infty} I_{i j}(t) \cos (\omega t) \mathrm{d} t}{\int_{0}^{\infty} I_{i j}(t) \mathrm{d} t}, \\
S_{i j}=\frac{\int_{0}^{\infty} I_{i j}(t) \sin (\omega t) \mathrm{d} t}{\int_{0}^{\infty} I_{i j}(t) \mathrm{d} t},
\end{gathered}
$$

where the subscripts $i j$ denote the coordinates of a given pixel, $\omega$ is the angular frequency related to the laser pulse repetition rate defined as $\omega=2 \pi f$, and $f$ here corresponds to $80 \mathrm{MHz}$. For a pixel characterized by a single-exponential decay, the phasor transform results in a set of coordinates that lies on the so-called universal semicircle described as

$(G-0.5)^{2}+S^{2}=0.25$.

For a fluorescence decay trace that contains two lifetimes, the phasor transform results in a coordinate pair that lies within the semicircle rather than on its boundary. The phasor coordinates, here, are determined by a linear combination of the two phasors corresponding to the two fluorescent species contributing to the signal. To compute the fluorescent lifetimes of a two-compound mixture, the eigenvector associated with the largest eigenvalue of the phasor cluster covariance matrix is commonly used to determine the orientation of the cluster's major axis. By extending this axis across the phasor plot, the short and long fluorescent lifetimes can be determined by the two intersections of the major axis with the universal semicircle. ${ }^{27,30}$

When there are three lifetimes, as expected in samples exposed to the sun filter formulation (sun filters: $120 \mathrm{ps,} \mathrm{free}$ NADH: 300 to $800 \mathrm{ps}$, and bound NADH: 1.0 to $6.5 \mathrm{~ns}$ ), the phasor coordinates are enclosed within a triangle whose vertices are located on the universal semicircle and correspond to the individual lifetimes of the three components. ${ }^{27}$ In the context of this study, the goal is to use phasor analysis to distinguish the NADH fluorescence (a compound with two lifetimes) from that of the chemical sun filters (a mixture with a third, distinct lifetime). To this aim, one method would be to compare the phasor coordinates of a given pixel to that of phasor clusters generated from (1) NADH alone and (2) sun filter formulation alone. This comparison could be quantified by computing the Euclidean distance between said pixel's phasor coordinates and those of the NADH and sun filter phasor cluster means. However, by strictly and only considering the mean coordinates of the NADH and sun filter phasors, all information relating to their distributions is essentially discarded.

Classically, NADH fluorescence lifetimes are typically thought of as either short or long, depending on whether the coenzyme is free or protein bound, respectively. However, recent work suggests that these protein-bound complexes can result in an entire subset of long lifetimes that are dependent on the nature of the protein to which NADH is bound. ${ }^{31,32}$ As a result, the phasor cluster corresponding to NADH fluorescence is fairly broad due to its multilifetime nature, warranting a 
separation technique that considers the statistical distributions of reference clusters for improved separation accuracy.

To this aim, the use of the Mahalanobis distance is proposed, where this metric describes the distance between a given observation and an entire distribution, rather than simply the distribution's mean. ${ }^{33}$ Mathematically, given an observation $x_{i j}=\left[G_{i j}, S_{i j}\right]^{\mathrm{T}}$ from a pixel with spatial coordinates $i j$ and a reference set with mean $\mu_{\text {ref }}=\left[G_{\mu}, S_{\mu}\right]^{\mathrm{T}}$ and covariance matrix $C_{\text {ref }}$, the Mahalanobis distance is defined as

$D_{i j, \text { ref }}=\sqrt{\left(\overrightarrow{x_{i j}}-\overrightarrow{\mu_{\text {ref }}}\right)^{\mathrm{T}} C_{\text {ref }}^{-1}\left(\overrightarrow{x_{i j}}-\overrightarrow{\mu_{\text {ref }}}\right)}$.

For a given pixel with spatial coordinates $i j$, the proportion $k_{i j}$ of photons that strictly arose from NADH as opposed to chemical sun filters is given by the ratio of its Mahalanobis distance to the sun filter mixture, $D_{i j, \mathrm{SF}}$, divided by the Mahalanobis distance from the NADH reference sample mean to the sun filter mixture, $D_{\mathrm{NADH}, \mathrm{SF}}$. If $k_{i j}$ is computed to be $<0.5$ (i.e., if more than half of the pixel's fluorescence can be attributed to the exogenous compound), then the perspective is flipped: the Mahalanobis distance from the phasor to the NADH reference cluster, $D_{i j, \mathrm{NADH}}$, is now divided by the Mahalanobis distance from the sun filter reference sample mean to the NADH reference cluster, $D_{\mathrm{SF}, \mathrm{NADH}}$. This ratio is then subtracted from 1 to provide the fraction of signal that arose from NADH as opposed to the sun filters. Mathematically, these relations can be expressed as

$$
k_{i j}=\frac{D_{i j, \mathrm{SF}}}{D_{\mathrm{NADH}, \mathrm{SF}}},
$$

if

$k_{i j}<\frac{1}{2}$

then

$k_{i j}=1-\frac{D_{i j, \mathrm{NADH}}}{D_{\mathrm{SF}, \mathrm{NADH}}}$.

By inspection, it is readily apparent that if a given phasor is very far from the sun filter phasor cluster, the computed proportion of photons generated from NADH approaches 1; conversely, as said phasor approaches the sun filter phasor cluster, the value of $k_{i j}$ approaches 0 . With this in mind, FLIM images containing fluorescence signals from both NADH and sun filters can be processed on a pixel-by-pixel basis to produce entire $k$-arrays whose elements are bound between 0 and 1 . The element-wise product of these arrays with the original TPEF intensity images can then be computed to isolate the fluorescence signal arising strictly from NADH. In turn, these processed images can be used in conjunction with FAD intensity images to compute optical redox ratios on a pixel-by-pixel basis to allow for quantification of oxidative stress in skin cells.

\subsection{Simulation and Validation}

To validate the improved accuracy of the proposed nonEuclidean approach over traditional Euclidean-based separation, simulated image data were generated and processed in MATLAB ${ }^{\circledR}$. First, endogenous and exogenous compounds were simulated to generate multivariate normally distributed random phasors with mean $\mu_{\text {endo }}=[0.42,0.35]^{\mathrm{T}}$ and covariance matrix $C_{\text {endo }}=10^{-4} \times[9,-2 ;-2,2.25]$ for the endogenous compound, and mean $\mu_{\text {exo }}=[0.96,0.05]^{\mathrm{T}}$ and covariance matrix $C_{\text {exo }}=10^{-5} \times[6.4,-1 ;-1,1.6]$ for the exogenous compound. The simulation then generates a set of three synthetic images $256 \times 256$ pixels in size: an endogenous reference image, an exogenous reference image, and a simulated test image.

For the test image, the concentration of endogenous compound is set to 1 on the far left pixel column and 0 on the far right, with a sigmoidal decay horizontally across the image. Conversely, the concentration of exogenous compound is 0 on the far left and 1 on the far right, with the sigmoidal decay in the opposite direction, such that the total compound content at any given pixel is 1 . The sigmoid describing the middle 254 columns is described by Eq. (6), where the column subscripts $j$ strictly range from 2 to 255 , inclusively

$$
k(j)=\frac{1}{1+\exp \left(\frac{j-128.5}{20}\right)} .
$$

The test image is then processed by the algorithm described in Sec. 2.5 using the simulated endogenous and exogenous images as NADH and chemical sun filter references, respectively. In addition, the test image is also processed using the classical Euclidean approach, where the endogenous fluorescence contribution is computed by dividing the Euclidean distance between the phasor and the exogenous reference by the sum of the Euclidean distances between the phasor and each reference image phasor cluster. ${ }^{29}$ The two analysis approaches are then compared by taking column-wise means to generate line plots, which intuitively illustrate the differences between the two methods from the expected simulated results.

\subsection{Quantification of Oxidative Stress}

To accurately calculate the fraction of fluorescence contributed by NADH as opposed to the chemical sun filters, the NADH images were first spatially binned using a mask $5 \times 5$ pixels in size. Only pixels with a minimum photon count of 1000 were considered for subsequent processing, where a custom script written in MATLAB ${ }^{\circledR}$ served to transform the data using the phasor approach and separate out the NADH fluorescence signal versus that of the chemical sun filters based on the non-Euclidean distance metric described in Sec. 2.5. Using the isolated fluorescence signal from NADH, NORR values for each field of view were then generated by computing FAD/(NADH + FAD) on a pixel-by-pixel basis. Given that both NADH and FAD are predominantly found within the cytoplasm and mitochondria with weaker fluorescence signals coming from nuclear regions, this correlated spatial distribution results in fairly consistent pixel-wise NORR values across the cell. As such, these pixel values can be averaged to obtain a single NORR value for each cell within a field of view. ${ }^{11,12,18}$ Individual cells were manually segmented from each resulting image using FIJI image analysis software. From there, mean NORR values were computed for each individual cell, from which an average NORR value could be computed for each individual field of view, representative of the cells present therein. The triplicate NORR values from all three imaged fields of view for each experimental condition were then used to compute a mean and a standard deviation. These triplicate measurements 
were then used for statistical comparison using the $\mathrm{R}$ software package for all statistical calculations. A one-way ANOVA was first calculated between all conditions, with the null hypothesis rejected. Pairwise student's t-test corrected using Holm's method was then carried out between groups to test for significance using $\mathrm{R}$ defaults. Results were considered statistically significance if $p<0.01$.

\section{Results and Discussion}

Beginning with the sun filter formulation alone, it is found to be transparent with no visible aggregates under transillumination at $755 \mathrm{~nm}$, as can be seen in Fig. 1(a); in Fig. 1(b), however, the formulation's fluorescence intensity in the NADH channel is readily notable. The corresponding phasor plot [Fig. 1(c)] shows the vast majority of the pixels forming a tightly bound cluster near the bottom right of the plot, highlighting its short lifetime. The formulation's fluorescence lifetime is in the order of $120 \mathrm{ps}$, as computed by fitting an exponential decay to the fluorescent decay trace of the sun filter mixture using SPCImage software [Fig. 1(d)].

An ex vivo examination of NADH fluorescence in the stratum spinosum of human epidermis at a depth of $50 \mu \mathrm{m}$ below the skin surface revealed bright signals from the cytoplasm, as $\mathrm{NADH}$ is located primarily in the mitochondria and cytosol
[Fig. 2(a)]. The corresponding phasor plot is shown in Fig. 2(b), where the NADH phasors cluster around the central upper region within the universal semicircle; the associated fluorescence decay of NADH is shown in Fig. 2(c). In the case of skin topically treated with the sun filter formulation, a coalesced pool of sun filter formulation can be appreciated in the bottom right portion of Fig. 2(d). In this scenario, the associated phasors are distributed between the original NADH cluster and the pure sun filter formulation cluster, as is made apparent by the elongated tail of the phasor plot in Fig. 2(e). Finally, Fig. 2(f) shows the decay trace of a pixel with a strong fluorescence contribution from the chemical sun filters.

The use of FLIM throughout the course of this study thus adds a temporal dimension over TPEF imaging alone, enabling the distinction of photons emitted by sun filters from those emitted by NADH using the algorithm described in Sec. 2.5 based on the Mahalanobis distance. To validate the accuracy of the developed method, simulated images of endogenous and exogenous references (i.e., NADH and chemical sun filters, respectively) as well as a test image were generated as described in Sec. 2.6. The phasor plots generated for the simulated endogenous reference, exogenous reference, and test sample are shown in Figs. 3(a)$3(\mathrm{c})$. The actual fluorescence contribution of the endogenous sample in the test image is shown in Fig. 3(d). Next, the
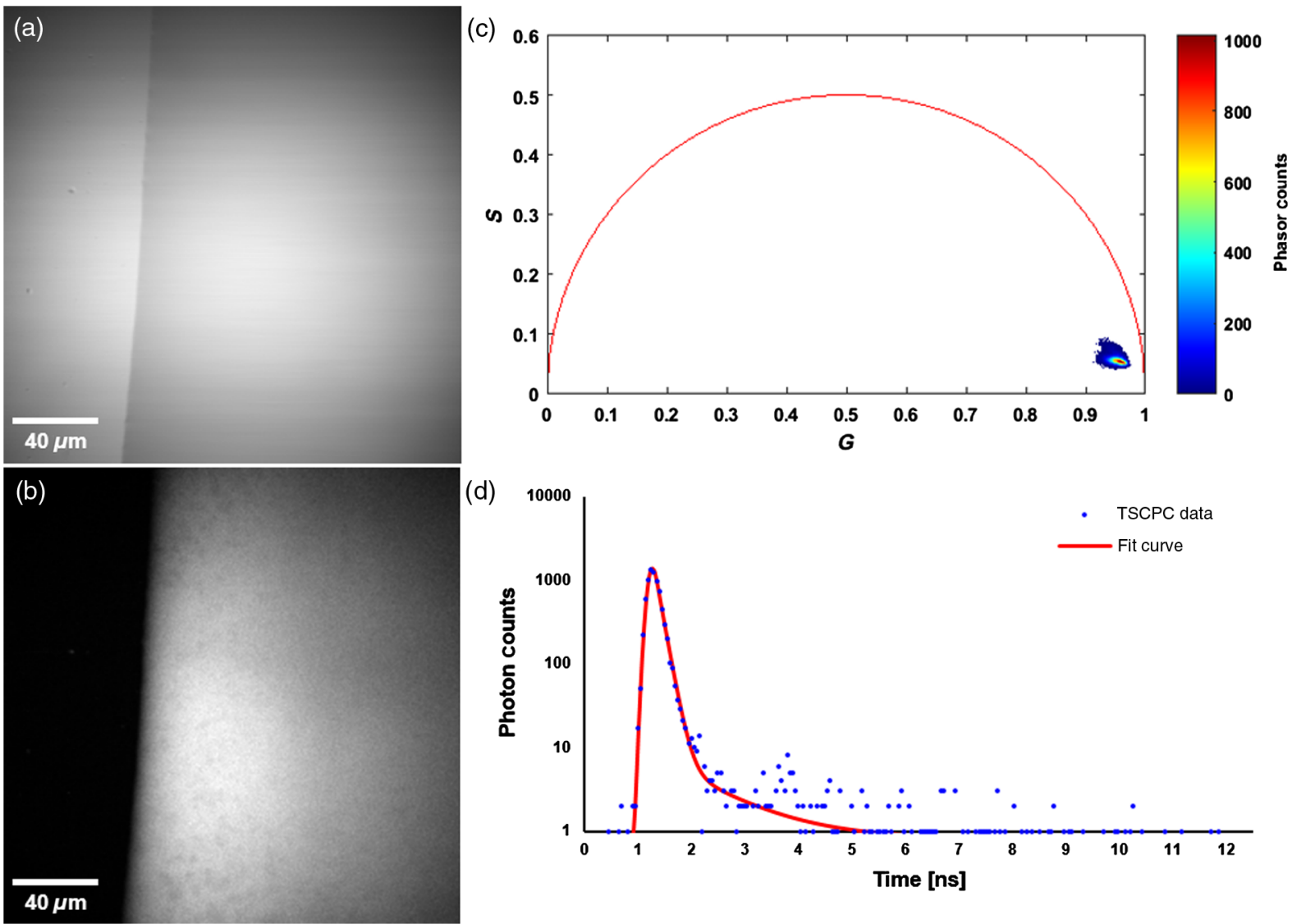

Fig. 1 Fluorescent properties of the sun filter formulation. (a) Transillumination image of the edge of a droplet of sun filter formulation, acquired with 755-nm light. (b) TPEF image of the sun filter formulation acquired with 755-nm excitation light and fluorescence detection from 445 to $480 \mathrm{~nm}$. (c) Phasor plot of the FLIM data associated with (b), highlighting the short fluorescence lifetime of the sun filter formulation. (d) Temporal decay trace of a typical pixel from (b), illustrating the rapid decay of the sun filter fluorescence. 

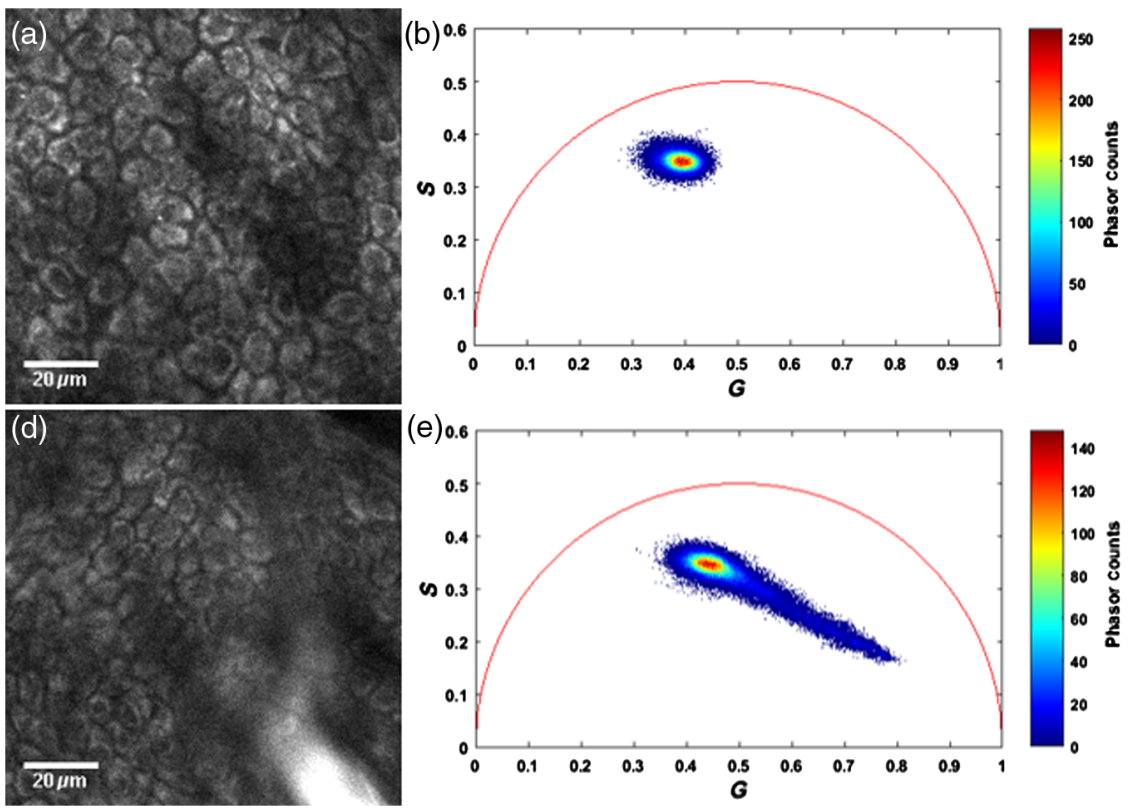
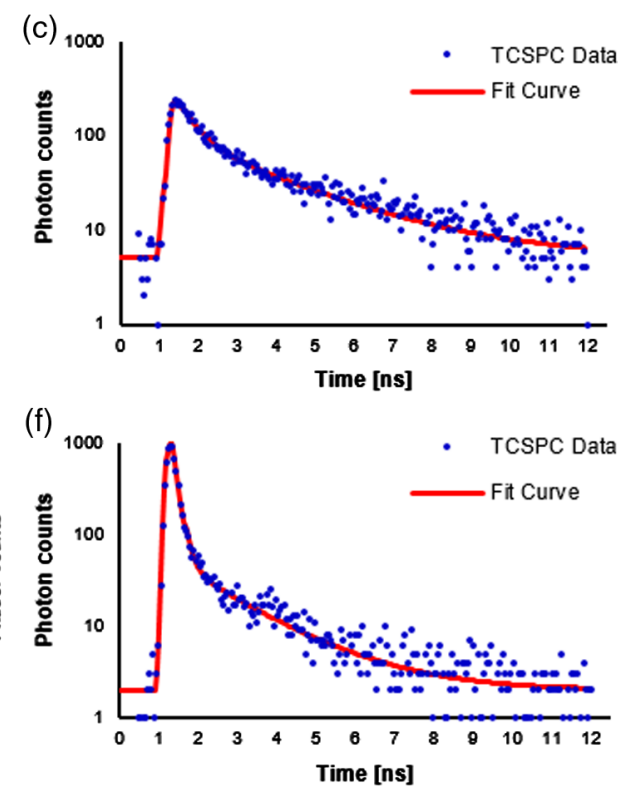

Fig. 2 Fluorescence detected from the stratum spinosum layer of the viable epidermis, acquired at a depth of $50 \mu \mathrm{m}$ below the skin surface. (a) TPEF image of human skin treated with vehicle only, showing endogenous NADH fluorescence. (b) Phasor plot of the FLIM data associated with (a). (c) Temporal decay trace of a pixel from (a), showing the progressive decay of NADH fluorescence. (d) TPEF image of human skin treated with sun filter formulation, showing both endogenous NADH fluorescence across the field of view, as well as exogenous fluorescence from the sun filters seen in the lower right region of the image. (e) Phasor plot of the FLIM data associated with (d). (f) Temporal decay trace of a pixel from (d) with a strong fluorescence contribution from chemical sun filters.

traditional Euclidean method [Fig. 3(e)] and the proposed non-Euclidean approach based on the Mahalanobis distance [Fig. 3(f)] are both used to estimate the endogenous fluorescence contribution. Finally, Fig. 3(g) shows the column-wise means of the endogenous fluorescence contribution images from Figs. 3(d)-3(f). While both methods accurately distinguish the two simulated fluorophores when their relative contributions are comparable, the traditional Euclidean method fails particularly in scenarios where one fluorophore with a broad phasor distribution dominates over another. This can be appreciated in the leftmost region of the curve in Fig. 3(g), where the Euclidean estimates plateau around 0.95 as the endogenous fluorescence begins to dominate, whereas the proposed Mahalanobis-based approach consistently follows the simulated data curve.

Having validated the proposed approach on simulated data, the algorithm was applied to all NADH image sets to decouple the endogenous fluorescence from that of the chemical sun filters. The performance of the algorithm is demonstrated in Fig. 4, showing the TPEF intensity image [Fig. 4(a)], the Euclidean estimate of the endogenous fluorescence contribution [Fig 4(b)], and the same estimate computed using the proposed non-Euclidean method [Fig. 4(c)]. Taken together, the results shown in Figs. 3 and 4 support the applicability of the described non-Euclidean separation algorithm for distinguishing endogenous NADH fluorescence from topically applied exogenous compounds with a similar fluorescence signature but a distinct lifetime.

While commercial sunscreens are typically formulated to remain in the uppermost layers of skin with minimal transdermal penetration, the FLIM data counterintuitively suggested that the chemical sun filters in the model formulation diffused throughout the viable epidermis. This diffusion into the skin is important to understand, as the pharmacokinetics of the sun filters will influence their oxidative effects.

The optimal design of topical formulations is challenging, as solvents/carriers and solutes may propagate throughout the tissue at different rates based on their charge, polarity, and structure. $^{13}$ While the imaging approach based on FLIM and phasor analysis developed here indeed demonstrates the ability to distinguish endogenous from exogenous fluorescence, coherent Raman imaging is a label-free modality that can be used to longitudinally visualize the diffusion of a solvent throughout the tissue by directly probing its molecular vibrational properties. ${ }^{34}$

Sunscreen formulations tend to be complex mixtures of diverse ingredients, whereas the model formulation studied here simply consisted of five chemical sun filters dissolved in Finsolv TN, a lipophilic solvent commonly used in sunscreen. Considering its molecular properties and the lipophilic nature of the sun filters, it was hypothesized that the solvent was acting as a carrier, thereby dragging the formulation contents into the deeper layers of the epidermis. To verify whether this was indeed the case, a $5-\mu \mathrm{L}$ droplet of Finsolv TN was topically applied to ex vivo human skin and visualized using CARS microscopy. CARS is a parametric four-wave mixing process where a known molecular vibrational resonance can be probed by interacting with two incident pulsed laser beams, known as the pump and Stokes beams, to generate a unique and spectrally distinct anti-Stokes signal that can be readily detected using an appropriate set of optical filters. ${ }^{35}$ In the case of Finsolv TN, the molecule has an alkyl tail 12 to 15 carbons in length; by probing the symmetric $\mathrm{CH}_{2}$ stretching vibrational mode at $2845 \mathrm{~cm}^{-1}$, the solvent can be readily visualized as it diffuses throughout the tissue. 
(a)

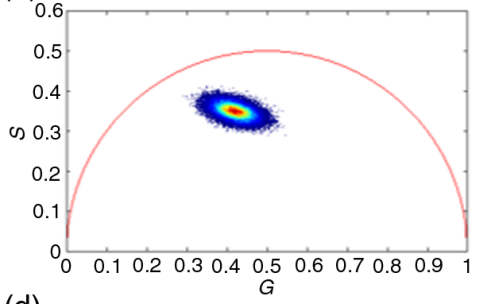

(d)

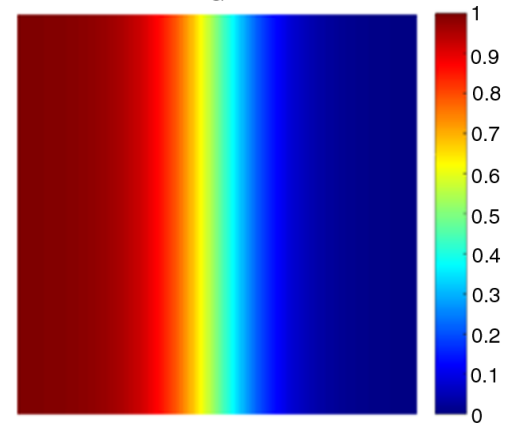

(b)

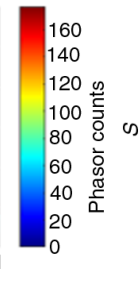

(e)
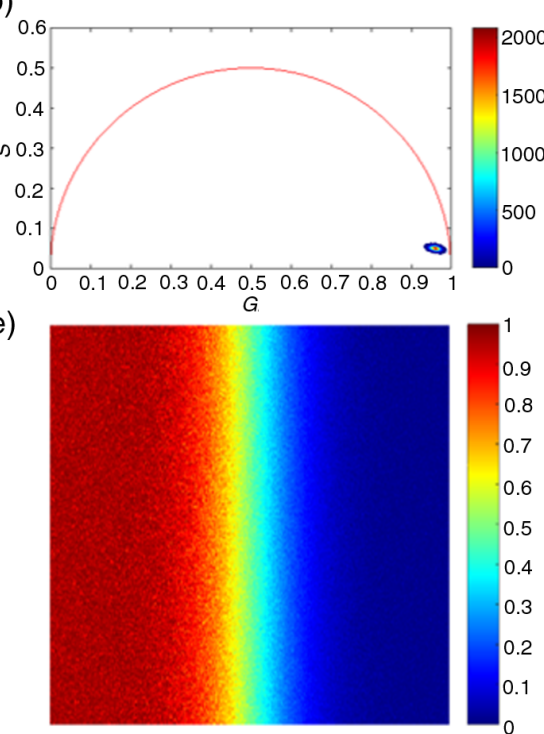

(c)

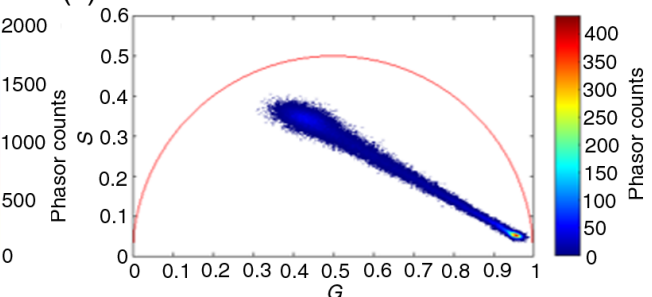

(f)

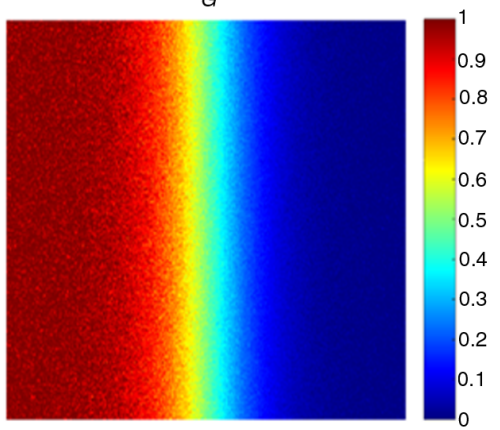

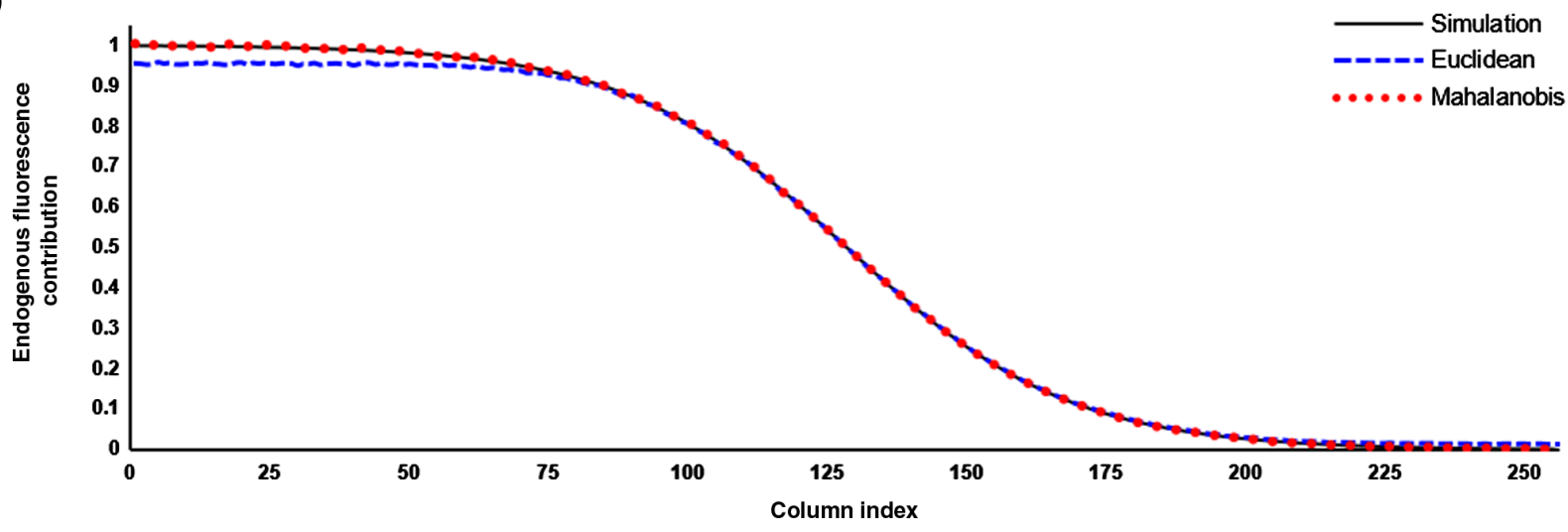

g)

Fig. 3 Simulation to validate the proposed non-Euclidean separation algorithm. (a) Phasor plot of simulated endogenous fluorescence reference sample. (b) Phasor plot of simulated exogenous fluorescence reference sample. (c) Phasor plot of simulated test image, consisting of both fluorophores with concentrations varying in opposite sigmoidal fashion. (d) Simulated endogenous fluorescence contribution to the test image. (e) Estimation of endogenous fluorescence contribution computed using the traditional Euclidean method. (f) Estimation of endogenous fluorescence contribution using the proposed nonEuclidean method based on the Mahalanobis distance. (g) Column-wise means of the images in (d-f), illustrating the superior accuracy of the proposed method over the classical Euclidean approach.
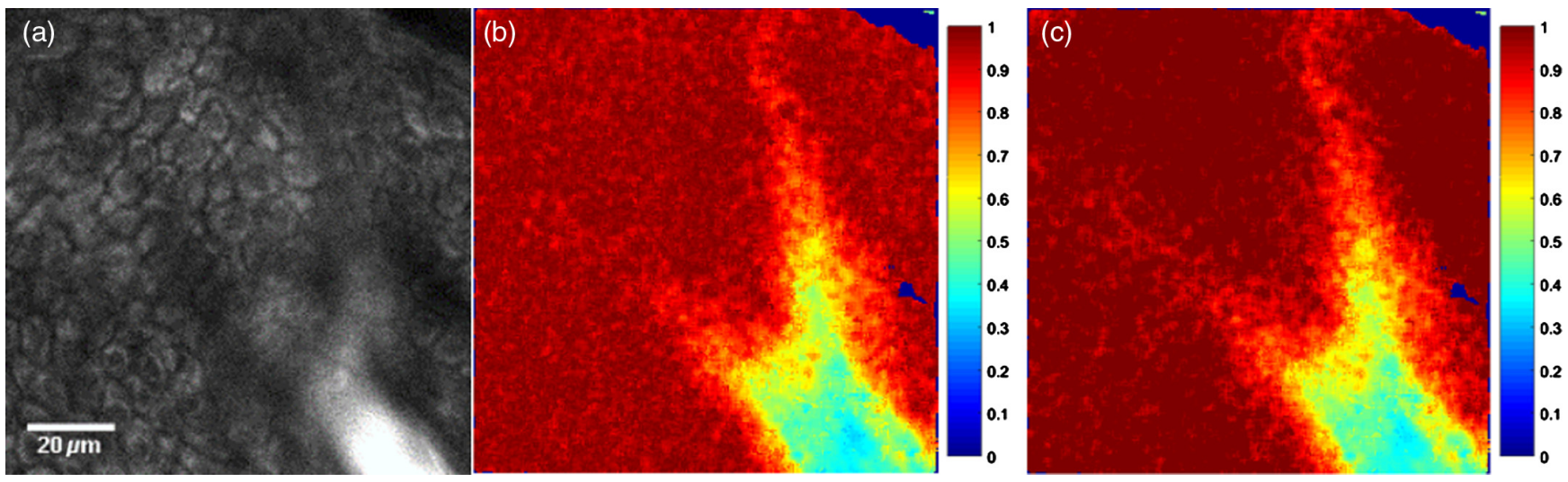

Fig. 4 Comparison of processing methods to optimally determine endogenous fluorescence contribution. (a) TPEF intensity image of human skin treated with chemical sun filters, showing a diffusing pool of formulation in the bottom right portion of the image. (b) Estimate of the endogenous fluorescence contribution computed using the traditional Euclidean method. (c) Estimate of the endogenous fluorescence contribution as determined by the proposed non-Euclidean approach based on the Mahalanobis distance metric. 

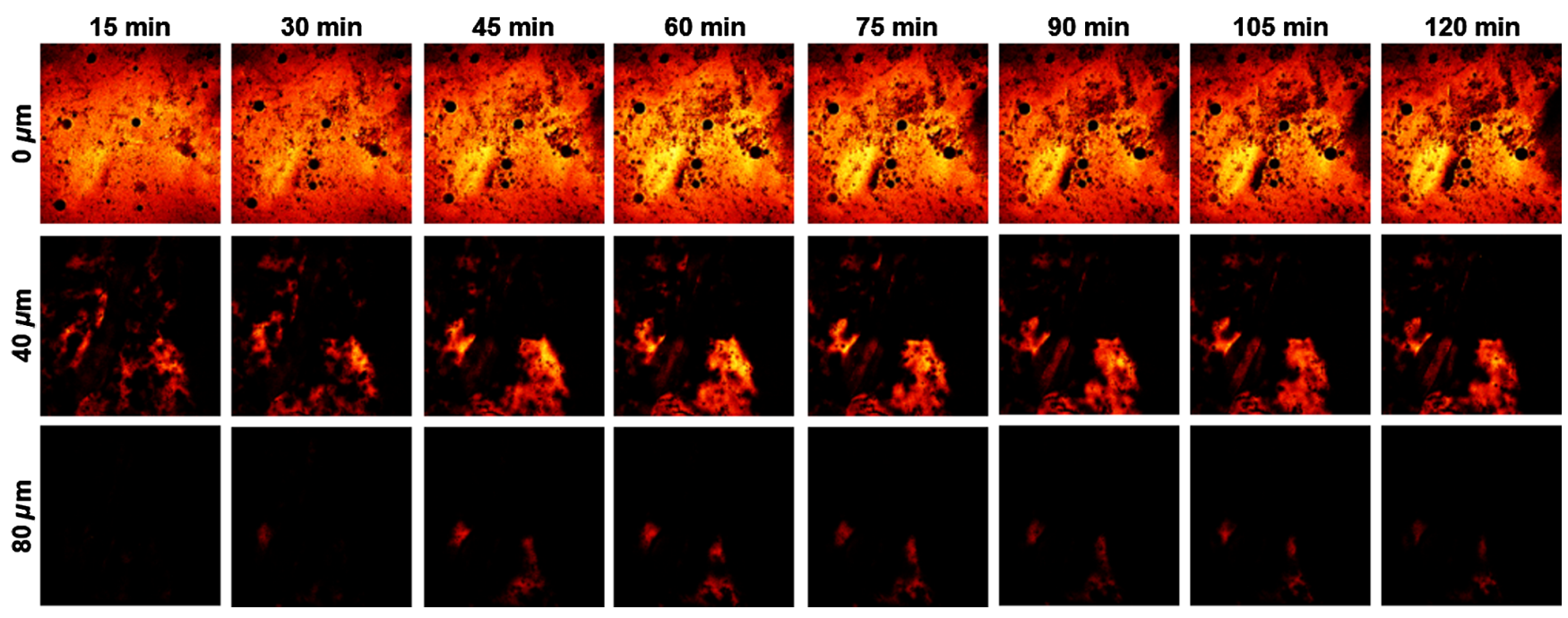

Fig. 5 Diffusion of the lipophilic solvent throughout various layers of human epidermis $(0,40$, and $80 \mu \mathrm{m}$ below the skin surface), as visualized using CARS microscopy to probe the $\mathrm{CH}_{2}$ vibrational mode of Finsolv TN at $2845 \mathrm{~cm}^{-1}$ at 15 -min intervals over the course of $2 \mathrm{~h}$. All images are $318 \mu \mathrm{m} \times$ $318 \mu \mathrm{m}$ in size.
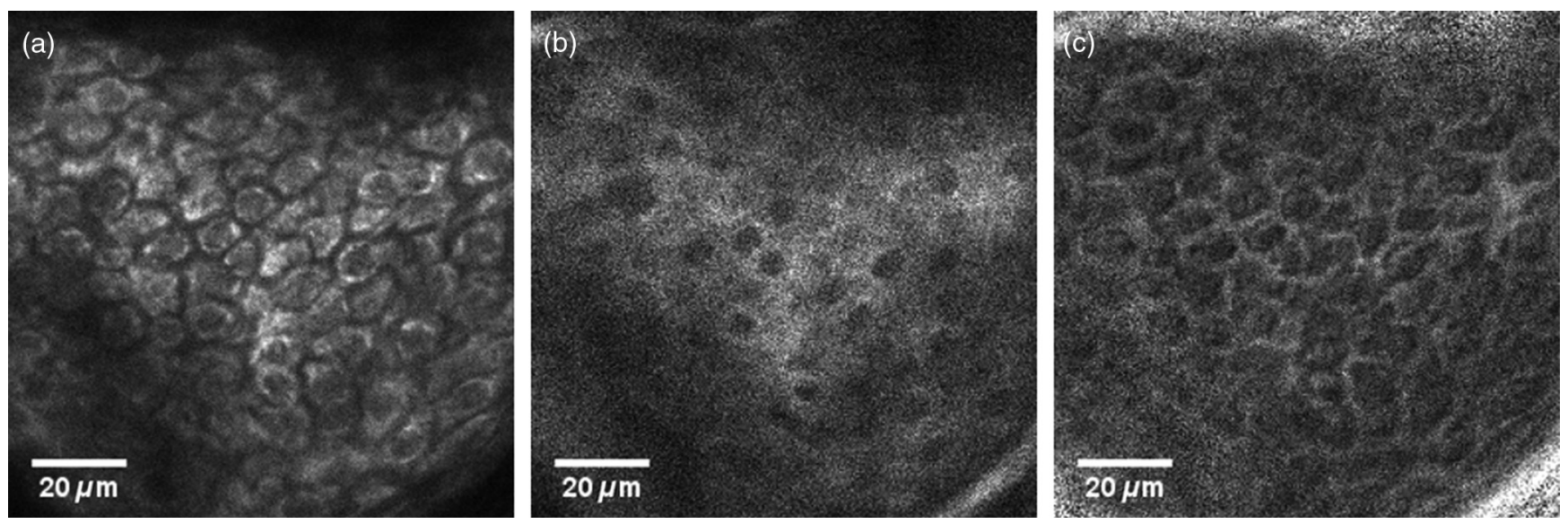

Fig. 6 Fluorescence intensity and computed NORR images of a typical skin sample. (a) NADH fluorescence intensity, (b) FAD fluorescence intensity, (c) NORR image, obtained by computing $\mathrm{FAD} /(\mathrm{NADH}+\mathrm{FAD})$ on a pixel-by-pixel basis.

As can be seen in Fig. 5, the solvent droplet shows bright CARS signals at the topmost layer of skin that progressively fades over time as the solvent diffuses and penetrates into the underlying strata. At 40 and $80 \mu \mathrm{m}$ below the surface, the intensity is seen to progressively rise, peak around 60 min following application, and fall thereafter as the Finsolv TN continues to diffuse radially throughout the epidermis. The use of CARS microscopy thereby confirmed the penetration of the formulation solvent into the deeper layers of the epidermis, consistent with the prior observation of sun filter fluorescence in the stratum spinosum using FLIM. More generally, this application of CARS imaging showcases the use of a distinct nonlinear optical modality for visualizing the formulation's solvent itself, which can prove useful in the optimization and validation of topical formulations, among other applications.

Having validated the penetration of and diffusion of both the sun filters and their solvent, we next studied whether these agents induce oxidative stress onto the cells of the viable epidermis. To this aim, NADH and FAD fluorescence intensities are used to compute NORR values, defined as FAD/(NADH + FAD), with a representative image set shown in Fig. 6.

This study's key finding is shown in Fig. 7, which shows the magnitude of redox perturbations in skin caused by both UVA and sun filter exposure. The greatest redox perturbation is a large oxidative shift triggered by exposure to UVA irradiation. This transition to an oxidative stress state is known to arise from the generation of ROS via UVA photochemistry and matches well what has been observed previously. $8,9,17,36-38$ Interestingly, we do indeed observe a small shift in redox state toward oxidative stress in skin tissues exposed only to the minimalist sun filter formulation $(p=0.08971)$, which would warrant further investigation with larger sample sizes. This oxidative shift is likely caused by the ROS generation mechanism previously described in in vitro studies, where the long tripletstate lifetime of the chemical sun filters enables them to react with ground-state triplet molecular oxygen, thereby promoting it to an excited singlet state. ${ }^{5,21,39-41}$ Importantly, however, when sun filter-treated skin was exposed to UVA irradiation, there is 


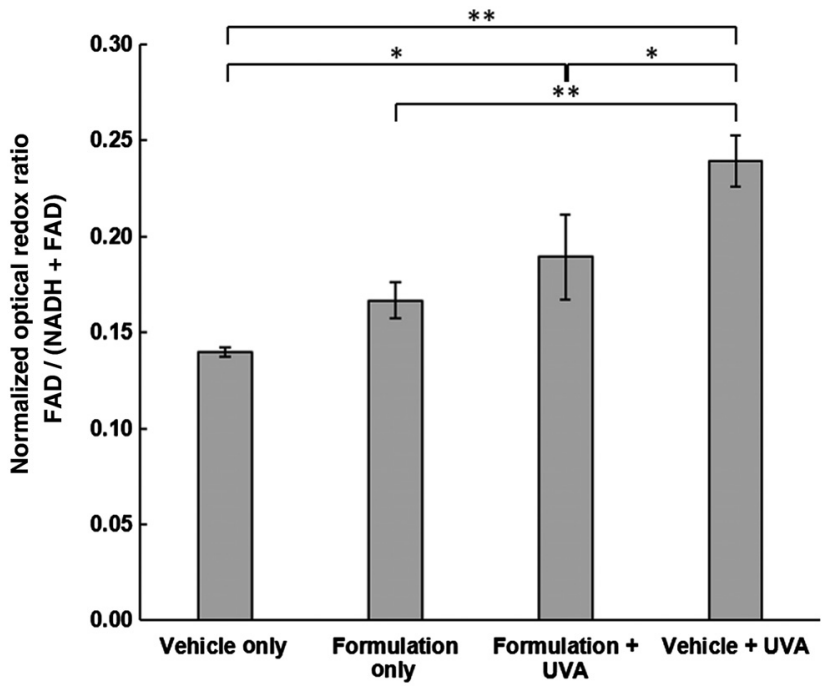

Fig. 7 NORR of cells in the stratum spinosum layer of the viable epidermis treated with either vehicle only or sun filter formulation and exposed to either 0 or $20 \mathrm{~J} / \mathrm{cm}^{2}$ (1 MED) of UVA irradiation. Bars correspond to mean NORR measurement of cells from $N=3$ fields of view, and error bars represent \pm 1 standard deviation. Statistical significance determined by pair-wise student's $t$-test corrected using Holm's method, annotated with * if $p<0.01$ and ${ }^{* *}$ if $p<0.005$.

a clear benefit, with the chemical sun filters limiting the oxidative stress induced by UV light exposure. This protection was observed even though a substantial concentration of the study's sun filter formulation was found to penetrate into the viable epidermis, indicating that these chemical absorbers still serve an important function even if they are not confined to the epidermal surface.

\section{Conclusions}

These results have important implications in the context of commercial sunscreen formulations and motivate the need for future studies investigating these effects. First, commercial sunscreens are purposely designed to limit the majority of skin penetration to the topmost layers of the epidermis; given that the formulation studied here primarily consisted of Finsolv TN, it is expected that the penetration of chemical sun filters observed in this context is not entirely representative of commercial sunscreen behavior. ${ }^{13}$ Second, sunscreens contain a wide variety of additional ingredients ranging from moisturizers to antioxidants, which could limit redox state perturbations, though the exact effects of these additives are not well-characterized in vivo. ${ }^{5,8,38}$ Still, the small redox changes observed due to the sun filter formulation begs the question as to what occurs following exposure to commercial products and what could be helpful in understanding how to best design topical sunscreens and sunscreen-containing cosmetics in the future.

It should be noted that while small oxidative shifts caused by the sun filters in this study were observed, the patient sample sizes in this preliminary work are small and are, therefore, not intended to support general claims regarding oxidative stress in vivo. Instead, this study describes a methodology that can now be expanded to investigate the effects of topical agents applied to skin in a noninvasive manner, in spite of the intrinsic fluorescence of the applied compounds. Specifically, it was found that the fluorescent nature of the studied chemical sun filters and, perhaps more importantly, their intrinsically short fluorescent lifetime, allow for spatiotemporal mapping of these compounds within skin. By exploiting the brevity of their fluorescence lifetime, the treated skin samples' time-correlated fluorescent emission from 445 to $480 \mathrm{~nm}$ can be transformed using the phasor approach to distinguish photons emitted from NADH versus chemical sun filters. This separation allows for analysis of redox state perturbations by processing the isolated NADH signals together with those of FAD to characterize the response of viable epidermal cells to chemical sun filter and/ or UVA exposure. In addition, this method enables the tracking of exogenous fluorescent compounds as they penetrate and permeate throughout the epidermis, as was shown in the present study with chemical sun filters, and further validated using CARS microscopy to monitor the solvent itself. As such, the proposed methodology can be applied to study a wide range of topical agents, provided the fluorescence lifetimes of the exogenous compounds are distinct from those of endogenous fluorophores.

This method is now ready for translation to clinical settings, where large-scale investigations into the kinetics and physiological dynamics of sun filters can be studied on human patients. Mobile two-photon FLIM systems are already commercially available and would be suitable for capturing the sun filters' diffusion and redox perturbations in skin. ${ }^{22,42}$ Importantly, future studies can focus on the use of more realistic commercial sun filter formulations to better inform the dermatology community, commercial sunscreen manufacturers, and consumers alike.

\section{Disclosures}

The authors declare no competing financial interests.

\section{Acknowledgments}

The authors acknowledge the members of the Evans group who have kindly agreed to provide constructive feedback on the paper. D.E.F. and C.L.E. acknowledge financial support from Johnson \& Johnson Inc. D.E.F. further acknowledges Grant support from the National Institutes of Health (Nos. 5R01 AR043369-19 and P01 CA163222) and the Dr. Miriam and Sheldon G. Adelson Medical Research Foundation.

\section{References}

1. J. K. Robinson, "Sun exposure, sun protection, and vitamin D," J. Am. Med. Assoc. 294(12), 1541-1543 (2005).

2. E. Thieden et al., "Sunscreen use related to UV exposure, age, sex, and occupation based on personal dosimeter readings and sun-exposure behavior diaries," Arch. Dermatol. 141(8), 967-973 (2005).

3. A. C. Green et al., "Reduced melanoma after regular sunscreen use: randomized trial follow-up," J. Clin. Oncol. 29(3), 257-263 (2011).

4. M. Huncharek and B. Kupelnick, "Use of topical sunscreens and the risk of malignant melanoma: a meta-analysis of 9067 patients from 11 casecontrol studies," Am. J. Public Health 92(7), 1173-1177 (2002).

5. K. M. Hanson, E. Gratton, and C. J. Bardeen, "Sunscreen enhancement of UV-induced reactive oxygen species in the skin," Free Radical Biol. Med. 41(8), 1205-1212 (2006).

6. J. Kockler et al., "Photostability of sunscreens," J. Photochem. Photobiol. C 13(1), 91-110 (2012).

7. C. Nishigori, Y. Hattori, and S. Toyokuni, "Role of reactive oxygen species in skin carcinogenesis," Antioxid. Redox Signaling 6(3), 561-570 (2004).

8. K. M. Hanson and C. J. Bardeen, "Application of nonlinear optical microscopy for imaging skin," Photochem. Photobiol. 85(1), 33-44 (2009).

9. M. Rinnerthaler et al., "Oxidative stress in aging human skin," Biomolecules, 5(2), 545-589 (2015). 
10. S. Q. Wang and H. W. Lim, Principles and Practice of Photoprotection, Springer, Switzerland (2016).

11. K. P. Quinn et al., "Characterization of metabolic changes associated with the functional development of 3D engineered tissues by noninvasive, dynamic measurement of individual cell redox ratios," Biomaterials 33(21), 5341-5348 (2012).

12. K. P. Quinn et al., "Quantitative metabolic imaging using endogenous fluorescence to detect stem cell differentiation," Sci. Rep. 3, 3432 (2013).

13. B. Godin and E. Touitou, "Transdermal skin delivery: predictions for humans from in vivo, ex vivo and animal models," Adv. Drug Delivery Rev. 59(11), 1152-1161 (2007).

14. I. Georgakoudi and K. P. Quinn, "Optical imaging using endogenous contrast to assess metabolic state," Аnnu. Rev. Biomed. Eng. 14, 351-367 (2012).

15. M. C. Skala et al., "In vivo multiphoton microscopy of NADH and FAD redox states, fluorescence lifetimes, and cellular morphology in precancerous epithelia," Proc. Natl. Acad. Sci. U. S. A. 104(49), 19494-19499 (2007).

16. A. A. Heikal, "Intracellular coenzymes as natural biomarkers for metabolic activities and mitochondrial anomalies," Biomarkers Med. 4(2), 241-263 (2010).

17. R. Datta et al., "Fluorescence lifetime imaging of endogenous biomarker of oxidative stress," Sci. Rep. 5, 9848 (2015).

18. C. A. Alonzo et al., "Two-photon excited fluorescence of intrinsic fluorophores enables label-free assessment of adipose tissue function," Sci. Rep. 6, 31012 (2016).

19. S. Q. Wang and H. W. Lim, "Current status of the sunscreen regulation in the United States: 2011 Food and Drug Administration's final rule on labeling and effectiveness testing," J. Am. Acad. Dermatol. 65(4), 863-869 (2011)

20. R. P. Stokes and B. L. Diffey, "The feasibility of using fluorescence spectroscopy as a rapid, non-invasive method for evaluating sunscreen performance," J. Photochem. Photobiol. B 50(2-3), 137-143 (1999).

21. A. Cantrell, D. J. McGarvey, and T. G. Truscott, "Photochemical and photophysical properties of sunscreens," Compr. Ser. Photosci. 3, 495519 (2001).

22. K. Koenig, "Hybrid multiphoton multimodal tomography of in vivo human skin," IntraVital 1(1), 11-26 (2012).

23. J. Xylas et al., "Noninvasive assessment of mitochondrial organization in three-dimensional tissues reveals changes associated with cancer development," Int. J. Cancer 136(2), 322-332 (2015).

24. A. D. Dunkelberger et al., "Picosecond dynamics of avobenzone in solution," J. Phys. Chem. A 119(24), 6155-6161 (2015).

25. T. B. Fitzpatrick, "The validity and practicality of sun-reactive skin types I through VI," Arch. Dermatol. 124(6), 869-871 (1988).

26. M. A. Pathak et al., "Sunlight and melanin pigmentation," Photochem. Photobiol. Rev. 1, 211-239 (1976).
27. M. A. Digman et al., "The phasor approach to fluorescence lifetime imaging analysis," Biophys. J. 94(2), L14-L16 (2008).

28. P. H. Lakner et al., "Applying phasor approach analysis of multiphoton FLIM measurements to probe the metabolic activity of three-dimensional in vitro cell culture models," Sci. Rep. 7, 42730 (2017).

29. M. Štefl et al., "Applications of phasors to in vitro time-resolved fluorescence measurements," Anal. Biochem. 410(1), 62-69 (2011).

30. C. Stringari et al., "Phasor fluorescence lifetime microscopy of free and protein-bound NADH reveals neural stem cell differentiation potential," PLoS One 7(11), e48014 (2012).

31. T. H. Chia et al., "Multiphoton fluorescence lifetime imaging of intrinsic fluorescence in human and rat brain tissue reveals spatially distinct NADH binding," Opt. Express 16(6), 4237-4249 (2008).

32. M. A. Yaseen et al., "Fluorescence lifetime microscopy of NADH distinguishes alterations in cerebral metabolism in vivo," Biomed. Opt. Express 8(5), 2368-2385 (2017).

33. P. C. Mahalanobis, "On the generalised distance in statistics," Proc. Natl. Inst. Sci. 2, 49-55 (1936).

34. C. W. Freudiger et al., "Label-free biomedical imaging with high sensitivity by stimulated Raman scattering microscopy," Science 322, 1857-1861 (2008).

35. C. L. Evans and X. S. Xie, "Coherent anti-stokes Raman scattering microscopy: chemical imaging for biology and medicine," Аппи. Rev. Anal. Chem. 1, 883-909 (2008).

36. R. M. Lavker et al., "Quantitative assessment of cumulative damage from repetitive exposures to suberythemogenic doses of UVA in human skin," Photochem. Photobiol. 62(2), 348-352 (1995).

37. S. Maleki et al., "Optical imaging of mitochondrial redox state in rodent model of retinitis pigmentosa," J. Biomed. Opt. 18(1), 016004 (2013).

38. K. M. Hanson and R. M. Clegg, "Observation and quantification of ultraviolet-induced reactive oxygen species in ex vivo human skin," Photochem. Photobiol. 76(1), 57-63 (2002).

39. J. M. Allen, C. J. Gossett, and S. K. Allen, "Photochemical formation of singlet molecular oxygen (1O2) in illuminated aqueous solutions of p-aminobenzoic acid (PABA)," J. Photochem. Photobiol. B 32(1-2), 33-37 (1996).

40. J. M. Allen, C. J. Gossett, and S. K. Allen, "Photochemical formation of singlet molecular oxygen in illuminated aqueous solutions of several commercially available sunscreen active ingredients," Chem. Res. Toxicol. 9(3), 605-609 (1996).

41. E. Gilbert et al., "Commonly used UV filter toxicity on biological functions: review of last decade studies," Int. J. Cosmet. Sci. 35(3), 208-219 (2013).

42. K. König et al., "Applications of multiphoton tomographs and femtosecond laser nanoprocessing microscopes in drug delivery research," Adv. Drug Delivery Rev. 63(4-5), 388-404 (2011).

Biographies for the authors are not available. 